\title{
An Advanced Genetic Optimization Algorithm to Solve Combined Economic and Emission Dispatch Problem
}

\author{
R. Gopalakrishnan and Dr.A. Krishnan
}

\begin{abstract}
The dispatch of electric load is one of the key functions in electrical power system operation, management and planning. The key intention of economic load dispatch is to reduce the total production cost of the generating system and at the same time the necessary equality and inequality constraints should also be fulfilled. In the present time, energy resources to generate mechanical power supplied to the rotor shaft of generating units are of fossil fuels. This leads to the emission of huge amount of carbon dioxide (CO2), sulfur dioxide (SO2) and nitrogen oxides (NOx) that results in atmospheric pollution. Reducing those pollutions resulted by usage of fossil-fired generating units has received great consideration. This provides wide field for the researchers to develop a better system to handle those needs. This leads to the development of Combined Economic and Emission Dispatch (CEED) techniques. There are various technique proposed by several researchers to solve CEED problem based on optimization techniques. The efficient optimization technique among the proposed work is Genetic Algorithm (GA). But still some problems like slower convergence and higher computational complexity exists in using GA for solving CEED problem. To overcome those difficulties, this paper uses Non- Dominated Ranked Genetic Algorithm (NRGA) which uses rank based Roulette Wheel selection algorithm with Pareto-based population ranking Algorithm. The simulation result shows that the proposed technique for solving combined economic and emission dispatch problem results in better convergence rate when compared to the existing techniques.
\end{abstract}

Keywords--- Combined Economic and Emission Dispatch (CEED), Genetic Algorithm (GA), Non-Dominated Ranked Genetic Algorithm (NRGA), Power Demand

\section{INTRODUCTION}

$\mathrm{T}$ HE economic dispatch difficulty has taken a appropriate twist as the public has turn out to be highly worried with environmental situations. The absolute minimum cost is not any more the only condition to be satisfied in the electric power generation and dispatching difficulties [18]. The production of electricity from the fossil fuel discharges various pollutants like Sulfur Oxides (SO2), and Oxides of

R. Gopalakrishnan, Assistant Professor, Department of Electrical and Electronics Engineering, KSR College of Engineering, Tiruchengode. E-mail: gopsengr@gmail.com

Dr.A. Krishnan, Dean, KS Rangasamy College of Technology, Tiruchengode.E-mail: amasikrishnan@hotmail.com
Nitrogen (NOX) into the atmosphere. These gaseous pollutants results in harmful effects on human beings and also on plants and animals. The Clean Air Act Amendments of 1990 (CAAA) suggest that the electricity using industry must decrease its $\mathrm{SO} 2$ emission by 10 million ton/year and the NOX by 2 million ton/year from the 1980 level.

On the other hand, considering only the operation of minimum environmental impact is not practical because of the high production cost of the system. Conversely, to operate the generating system with the lesser production cost will result in higher emission. As a result, economic dispatch, emission dispatch or combined economic and emission dispatch [15] is in some way selected separately or combined together. To determine the suitable solution to this difficulty, an excellent power management approach is set. Various optimization methods like lambda iteration, linear programming, non-linear programming, quadratic programming, interior point technique or even intelligent search techniques (e.g. Genetic Algorithm (GA), Evolutionary Programming (EP), Particle Swarm Optimization (PSO), etc) are used to overcome several economic dispatch difficulties and also the unit commitment difficulties.

The usage of genetic algorithm for economic dispatch [12, $16,17]$ difficulties needs a large number of generations if the power generating system has the more number of units. Combined economic and emission dispatch [20,22] has been emerged in the field of power generation dispatch [19] that concurrently reduce both fuel cost and total emissions. While the emission is reduced the fuel cost may be inappropriately increased or while the fuel cost is reduced the emission may be increased. This difficulty is overcome by creating the objective function and utilizing some optimization methods like PSO and GA. But, there are some problems like more time for optimization, operation complexity, etc., exists in using those optimization techniques. To overcome those difficulties, this paper uses Non-Dominated Ranked Genetic Algorithm (NRGA). The remaining sections of this paper will explain the methodology and the result obtained using the proposed technique to solve CEED problem $[11,13,14]$.

\section{RELATED WORKS}

A novel form of dynamic programming method is proposed by Muralidharan et al., [1]. The main aim of this approach is that cost, emission and loss are combined and moreover the pareto-optimal economic dispatch for emission constrained and loss-restricted case. In this approach, a novel Dynamic Programming (DP) approach replaces the 
conventional optimization approaches. It's a new recursive technique for recognizing production cost minimization, with an emission constrained and loss reduced condition. DP is a mathematical approach which deals with the optimization of multistage decision process. A final operating condition with minimum production cost at reduced emission rate while maintaining stability leads to a multi-objective problem which is successfully carried out in this approach.

Prasanna et al., [2] proposed Fuzzy-Tabu search algorithm for combined economic and emission dispatch. Combined Economic Emission Dispatch (CEED) issue is to schedule the committed generating unit outputs to satisfy the needed load demand at minimum operating cost with minimum emission simultaneously. This multi-objective CEED problem [24, 25] is changed into a single objective function with the help of a price penalty factor. Ugur Guvenc et al., [3] proposed a novel Genetic Algorithm technique based on similarity crossover for solving CEED problem in power systems. Senthil et al., [4] uses Improved Tabu Search Algorithm (ITS) which minimizes the cost per unit power consumption. Lakshmi Devi et al., [5]proposes a lambda based technique for solving the CEED problem using Genetic Algorithm (GA) and Particle Swarm Optimization (PSO) techniques considering the power limits of the generator. The proposed technique finds the global or near-global optimal solution for the Combined Economic and Emission Dispatch (CEED) problem.

Lakhwinder Singh et al., [6] deals with the Economic Emission Dispatch (EED) issue involving real and reactive power scheduling of thermal power generating units. Demirel et al., [7] proposed Economic and minimum emission dispatch with Hopfield Neural Network (HNN) [10, 23] and Lagrange Multiplier (LM) solutions to economic dispatch (ED), $\mathrm{NO}_{\mathrm{x}}$ Emission Dispatch (EmD), and Economic-Emission Dispatch (EED) [9] of a sample system which contains six thermal generators are presented. T. Ratniyomchai et al., [8] presents a demonstration of solving combined economic and emission dispatch problems. The main aim of the combined problem can be represented by considering both the fuel cost and total emission with necessary constraints. Particle Swarm Optimization (PSO) [26] is one of most efficient intelligent search techniques involved in solving economic load dispatch. PSO is exploited in this approach to demonstrate its significance. The two economic and emission dispatch problems are combined and converted into a single objective function $[20,21]$ with the help of a price penalty factor $h$. The converted objective function is minimized based on efficient PSO.

\section{METHODOLOGY}

\section{A. Economic Dispatch}

The economic dispatch problem is to determine the optimal mixture of power generation in a manner that the entire production cost of the entire system is reduced while satisfying the total power demand and few key power system factors. The fuel cost for all the power generation unit is defined initially. Therefore, the total production cost function of economic dispatch problem is defined as the total sum of the fuel costs of all the generating plant units as mentioned below:

$$
F_{T}=\sum_{i=1}^{N_{G}}\left\{a_{i} P_{i}^{2}+b_{i} P_{i}+c_{i}+\left|d_{i} \sin e_{i}\left(P_{i}^{\min }-P_{i}\right)\right|\right\}
$$

Where

$N_{G}$ is the total number of generating units

$F_{T}$ is the total production cost

$P_{i}$ is the power output of generating unit $i$

$P_{i}^{\text {min }}$ is the minimum output of generating unit $i$

$a_{i}, b_{i}, c_{i}, d_{i}, e_{i}$ are fuel cost coefficients of unit $i$

This equation helps in determining the total production cost of the generating plant.

\section{B. Emission Dispatch}

The solution of economic dispatch problem will provide the quantity of active power to be produced by various units at the minimum production cost for a certain power requirement. On the other hand, the total quantity of pollutant emission is not considered in conventional economic dispatch problem. The quantity of pollutant emission resulted from a fossil-fired thermal generating unit is based on the amount of power generated by every unit. For reducing the complexity, the total emission produced can be modeled as a direct sum of a quadratic function and an exponential term of the active power output of the generating units. The pollutant emission dispatch problem can be described as the optimization of total amount of pollutant emission given as below:

$$
E_{T}=\sum_{i=1}^{N_{G}}\left\{\alpha_{i} P_{i}^{2}+\beta P_{i}+\gamma_{i}+\xi_{i} e^{\tau_{i} P_{i}}\right\}
$$

Where

$N_{G}$ is the total number of generating units

$F_{T}$ is the total pollutant emission

$P_{i}$ is the power output of generating unit $i$

$\alpha_{i}, \beta_{i,}, \gamma_{i,}, \xi_{i,}, \tau_{i}$, are emission coefficients of unit $i$

\section{Combined Economic and Emission Dispatch}

The economic dispatch and emission dispatch are two various problems as discussed previously. Emission dispatch can be included in conventional economic load dispatch problems by merging an emission constraint with the economic load dispatch problem. In this paper, the two objectives can be converted into a single objective function by introducing a price penalty factor as defined follows.

$$
h=\frac{F_{T}\left(P_{i}^{\max }\right) / P_{i}^{\max }}{E_{T}\left(P_{j}^{\max }\right) / P_{j}^{\max }}
$$


Where

$h$ is the price penalty factor

$i$ is the highest fuel-cost unit

$j$ is the highest pollutant-emission unit

The combined objective function of the economic and emission dispatch is represented by the following equation:

$$
\Phi_{T}=w_{e c o} F_{T}+w_{e m i} h E_{T}
$$

Where

$\Phi_{T}$ is the combined objective function

$w_{\text {eco }}, w_{\text {emi }}$ are weighting factors.

The two weighting factors can be provided in various ways. The case of $w_{\text {eco }},=1.0$ and $w_{\text {emi }}=0.0$ is to obtain the classical economic dispatch problem and the pure emission dispatch is resulted when $w_{\text {eco }},=0.0$ and $w_{\text {emi }}=1.0$. To obtain the combined economic and emission dispatch problem, both weighting factors should be equal, for example $w_{e c o},=0.5$ and $w_{e m i}=0.5$.

\section{Problem Constraints}

Usually, there are two constraints such as equality and inequality constraints should be considered. For the problem defined in this paper, a power balance equation (5) is set as an equality constraint and the limits of power generation output (6) are set as inequality constraints.

$$
\begin{gathered}
P_{D}+P_{\text {Loss }}-\sum_{i=1}^{N_{G}} P_{i}=0 \\
P_{i}^{\text {min }} \leq P_{i} \leq P_{i}^{\text {max }}, i=1,2, \ldots, N_{G}
\end{gathered}
$$

Where

$P_{D}$ is the total power demand of the plant

$P_{\text {Loss }}$ is the total power losses of the plant

$P_{i}^{\text {min }}$ is the minimum output of generating unit $i$

$P_{i}^{\max }$ is the maximum output of generating unit $i$

With this defined problem and objective function, an appropriate optimization technique is used to obtain the required objective. This paper uses Non-Dominated Ranked Genetic Algorithm as optimization technique which has various advantages over the existing system which uses genetic algorithm to solve the CEED problem.

\section{E. Genetic Algorithm}

The Genetic Algorithm (GA) is an optimization and stochastic global search technique based on the principles of genetics and natural selection. A GA permits a population composed of several individuals to develop under particular selection rules to a state that reduces the fitness (i.e., minimizes the cost function). The technique was developed by John Holland (1975) over the course of the 1960s and 1970s and finally popularized by one of his student, David Goldberg (1989).

Optimization of a problem in a Genetic Algorithm is realized within the fitness function. As the proposed technique utilizes the equal incremental cost criterion as its basis, the constraint equation can be rewritten as:

$$
\varepsilon=\left|\sum_{i=1}^{N_{G}} P_{i}-P_{D}-P_{\text {Loss }}\right|
$$

Then, the converging rule is when $\varepsilon$ (error) decreases to within a particular value. For the purpose of emphasizing the "best" chromosomes and speed up convergence of the iteration procedure, fitness is normalized into the range between 0 and 1. The fitness function used is:

$$
F I T=\frac{1}{1+k\left(\frac{\varepsilon}{P_{D}}\right)}
$$

Where, $\mathrm{k}$ is a scaling constant.

In the discrete Genetic Algorithm, resulting point is a binary string of 0 and 1 called chromosome and number of bits (Nbits) is based on preferred accuracy. The string is constructed of $n$ variables $(\mathrm{x} 1, \mathrm{x} 2, \ldots, \mathrm{xn})$, therefore the number of bits for every variable is Nbits/n called "gene". A sample solution point with 8-bits and two variables $(x, y)$ is provided below:

$$
\begin{array}{llll}
1 & 0 & 1 & 1\} \text { gene } 1(X) \\
0 & 0 & 0 & 1\} \text { gene2 }(Y)
\end{array}
$$

The initial 4 bits are associated to $\mathrm{x}$ and next 4 bits are associated to y. To compute the cost of solution points, they should be decoded initially. Decoded structure of the mentioned string is computed as below:

$$
\begin{gathered}
\left\{\begin{array}{c}
x \rightarrow 1 \times 2^{3}+0+1 \times 2^{1}+1 \times 2^{0}=11 \\
y \rightarrow \quad y \rightarrow 0+0+0+1 \times 2^{0}=1
\end{array}\right. \\
=\operatorname{Cost}(11,1)
\end{gathered} \Rightarrow \operatorname{Cost}(x, y)
$$

\section{A: The population}

The GA begins with a collection of chromosomes known as the population. The population has Npop chromosomes called population size.

\section{B: Natural selection}

Natural selection is carried out on the population by maintaining the most capable individuals according to their fitness. In this manner, it is likely to keep the size of the population constant, for convenience. Inititally, the Npop costs and associated chromosomes are ranked in order that lowest 
cost comes first and then comes the highest cost. Next, only the best chromosomes are chosen to persist, whereas the remaining is neglected. The selection rate, Xrate, is the fraction of Npop that stays alive for the next step of mating (crossover). The number of chromosomes that are maintained in every generation is:

\section{C: Selection}

$$
N_{\text {keep }}=X_{\text {rate }} \times N_{\text {pop }}
$$

For the purpose of replacing the neglected chromosomes and maintain the population size constant, two chromosomes are chosen from the mating pool of Nkeep chromosomes to generate two new offspring. Pairing process is performed in the mating population until Npop-Nkeep offspring are generated to substitute the neglected chromosomes.

\section{D: Crossover}

Mating is nothing but the creation of one or more offspring from the parents chosen in the pairing process. The present members of the population limit the genetic composition of the population. The most common form of mating engages two parents that produce two offspring.

\section{E: Mutation}

Random mutations modify some percentage of the bits in the list of chromosomes. Mutation is the second technique a Genetic Algorithm explores a cost surface. It can introduce traits not in the original population and keeps the GA from converging too fast before sampling the entire cost surface.

Limitations of Genetic Algorithm in CEED problems are

- Slow convergence

- It lacks rank based fitness function which reduces complexity

So the proposed approach uses the Non Dominated Ranked Genetic Algorithm (NRGA) for the optimization purpose. The main advantages of using Non Dominated Ranked Genetic Algorithm are that it converges very significantly than GA. Moreover, it is provides rank based fitness function and it is quicker than GA.

\section{F. Non-Dominated Ranked Genetic Algorithm}

At first, a random parent population $\mathrm{P}$ is formed. The sorting of the population is in accordance with the nondomination. Every solution is allocated a fitness (or rank) equivalent to its non-domination level. Non-domination level of 1 represents the best level, 2 represents the next-best level, etc.

Therefore, minimization of fitness is implicit. Initially, the normal Ranked accorded Roulette wheel choosing, recombination, and mutation operators are applied to generate an offspring population Q of size N. As elitism is initiated by contrasting present population with earlier obtained best nondominated results, the process is varied after the starting generation. Initially the ith generation of the presented algorithm as shown in below is explained.

The algorithm represents that Non-Dominated Ranked Genetic is simple and straightforward. Initially, a combined population PUQ is created. The mixed population is of size $2 \mathrm{~N}$ then obtained; the mixed population is sorted based on the non-domination. As every previous and present population members are incorporated in the mixed population elitism is guaranteed. This process will choose $\mathrm{N}$ solutions out of $2 \mathrm{~N}$.

The new population of size $\mathrm{N}$ is utilized for choosing. Next, two tiers ranked dependent roulette wheel selection is used, one tier to choose the front and the other to choose solution from the front, here the results obtained for the finest nondominated set F1 have the higher probabilities to be chosen. Therefore, results from the set F2 are selected with small probability than results from the set F1 and so on. After that crossover and mutation are used to generate a new population $\mathrm{P}$ of size $\mathrm{N}$. The diversity between non-dominated results is established by the second tier of ranked dependent roulette wheel selection that ranks the results according to their crowding distance. The results with lesser crowding distance will have the higher probabilities.

As solutions contend with their crowding distance, no extra niching attribute is needed. Even though the crowding distance is computed in the objective function space, it can also be obtained in the parameter space, if required. The NRGA algorithm is shown in figure 2.

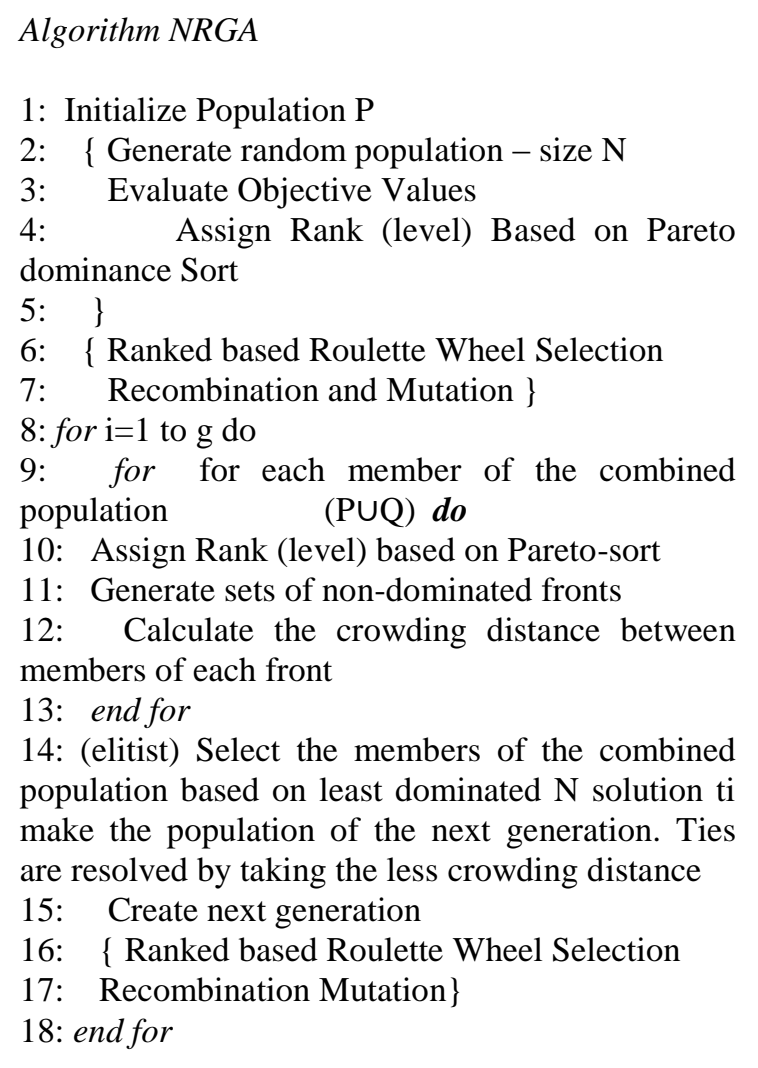

Figure 1: Non-Dominated Ranked Genetic Algorithm

Finally, the optimized parameters will help in achieving the solution for combined economic and emission dispatch problem. The evaluation of the proposed technique for combined economic and emission dispatch is presented in the simulation results. 


\section{Simulation Results}

The proposed method for combined economic and emission dispatch problem is evaluated using the six generator system. Fuel cost coefficients and generation limits for each generating unit of the test system were given in table 1 and the emission coefficient is given in table 2. The proposed technique is tested by varying the power demand such as 400 MW, $500 \mathrm{MW}, 600 \mathrm{MW}, 700 \mathrm{MW}, 800 \mathrm{MW}, 900 \mathrm{MW}$ and $1000 \mathrm{MW}$. The resulted CEED solution for the considered sixgenerator system is presented in table 3 . From the table, it can be observed that the cost required for generating the required power increases as the power demand rises. Also, the emission output rises as the power demand rises.

The fuel cost required for particular power demand and the resulted emission output by using the proposed technique can be found in table 3. The proposed technique is evaluated with the results of using optimization techniques like Particle Swarm Optimization (PSO) and Genetic Algorithm (GA). The fuel cost required by various techniques is provided in table 4 . From the result, it can clearly suggest that the proposed technique is better which required lesser cost for the system operation when compared to other techniques.
The emission resulted for using PSO, GA and NRGA for various power demands is provided in table 5. The emission resulted for using the proposed optimization technique is very much reduced when compared to the other techniques. This case is true not only for particular power demand rather it is true for all cases.

The number of iterations required for optimization using PSO, GA and NRGA is provided in table 6 and figure 3. It can be observed that the proposed technique required lesser iterations for optimization when compared to other techniques. Also, the time required for optimization is much reduced for the proposed technique when compared to other techniques. It is represented in table 7 and figure 4.

By considering the overall result, it can be suggested that the usage of proposed technique will reduce the fuel cost as well as the emission output. Also, the time required by the proposed technique is much reduced when compared to the other conventional techniques.

Table 1: Fuel Cost Coefficients for Six-Generator System

\begin{tabular}{|c|c|c|c|c|c|c|c|}
\hline Generator & $\mathrm{a}$ & $\mathrm{b}$ & $\mathrm{c}$ & $\mathrm{d}$ & $\mathrm{e}$ & $\mathrm{P}_{\min }$ & $\mathrm{P}_{\max }$ \\
\hline 1 & 100 & 200 & 12 & 10 & 8.45 & 10 \\
\hline 2 & 110 & 190 & 15 & 20 & 11.45 & 15 \\
\hline 3 & 115 & 210 & 10 & 10 & 14.87 & 18 \\
\hline 4 & 90 & 195 & 10 & 15 & 17.54 & 200 \\
\hline 5 & 105 & 180 & 20 & 10 & 16.69 & 220 \\
\hline 6 & 120 & 170 & 15 & 20 & 20.21 & 250 \\
\hline
\end{tabular}

Table 2: Emission Coefficients for Six-Generator System

\begin{tabular}{|c|c|c|c|c|c|}
\hline Generator & $\alpha$ & $\beta$ & $\gamma$ & $\xi$ & $\tau$ \\
\hline 1 & 5.743 & -5.076 & 3.870 & $2 \times 10^{-4}$ & 4.678 \\
\hline 2 & 5.194 & -5.987 & 4.765 & $3 \times 10^{-4}$ & 3.034 \\
\hline 3 & 6.542 & -4.054 & 4.353 & $3 \times 10^{-4}$ & 7.361 \\
\hline 4 & 5.764 & -5.875 & 5.769 & $4 \times 10^{-4}$ & 5.908 \\
\hline 5 & 7.109 & -6.045 & 3.812 & $1 \times 10^{-6}$ & 6.210 \\
\hline 6 & 5.431 & -5.342 & 4.873 & $2 \times 10^{-4}$ & 4.890 \\
\hline
\end{tabular}

Table 3: CEED Solution of Six-Generator System

\begin{tabular}{|c|c|c|c|c|c|c|c|}
\hline & \multicolumn{7}{|c|}{ Power Demand $(M W)$} \\
\cline { 2 - 8 } & 400 & 500 & 600 & 700 & 800 & 900 & 1000 \\
\hline Unit 1 & 15.01 & 19.21 & 24.26 & 39.54 & 45.19 & 65.21 & 69.21 \\
\hline Unit 2 & 19.21 & 28.35 & 35.75 & 65.31 & 74.18 & 91.56 & 103.51 \\
\hline Unit 3 & 51.09 & 65.05 & 71.51 & 96.09 & 102.43 & 128.45 & 135.43 \\
\hline Unit 4 & 89.11 & 103.96 & 135.34 & 149.62 & 161.51 & 175.97 & 187.41 \\
\hline
\end{tabular}




\begin{tabular}{|c|c|c|c|c|c|c|c|}
\hline Unit 5 & 98.07 & 124.33 & 147.23 & 161.32 & 194.57 & 208.37 & 228.85 \\
\hline Unit 6 & 127.51 & 159.10 & 185.91 & 188.12 & 222.12 & 230.44 & 275.59 \\
\hline $\begin{array}{c}\text { Fuel Cost } \\
\mathbf{( \$ / h )}\end{array}$ & 28564.87 & 34674.12 & 37912.65 & 42632.98 & 47923.64 & 52543.76 & 59721.54 \\
\hline $\begin{array}{c}\text { Emission } \\
\text { Output } \\
\text { (Kg/h) }\end{array}$ & 253.34 & 301.56 & 405.54 & 521.56 & 645.56 & 721.87 & 879.34 \\
\hline
\end{tabular}

Table 4: Required Fuel Cost $(\$ / h)$ for using Different Techniques

\begin{tabular}{|c|c|c|c|c|c|c|c|}
\hline \multirow{2}{*}{$\begin{array}{c}\text { Optimization } \\
\text { Technique }\end{array}$} & \multicolumn{7}{|c|}{ Power Demand $(M W)$} \\
\cline { 2 - 8 } & 400 & 500 & 600 & 700 & 800 & 900 & 1000 \\
\hline PSO & 28567.11 & 34676.45 & 37915.11 & 42635.23 & 47925.23 & 52545.35 & 59724.12 \\
\hline GA & 28565.24 & 34675.04 & 37913.54 & 42634.23 & 47924.45 & 52544.81 & 59724.32 \\
\hline NRGA & 28564.87 & 34674.12 & 37912.65 & 42632.98 & 47923.64 & 52543.76 & 59721.54 \\
\hline
\end{tabular}

Table 5: Emission Output ( $\mathrm{kg} / \mathrm{h})$ for using Different Techniques

\begin{tabular}{|c|c|c|c|c|c|c|c|}
\hline $\begin{array}{r}\text { Power Demand } \\
(\mathrm{MW})\end{array}$ & 400 & 500 & 600 & 700 & 800 & 900 & 1000 \\
$\begin{array}{r}\text { Optimization } \\
\text { Technique }\end{array}$ & 254.89 & 303.98 & 406.78 & 524.98 & 647.91 & 723.17 & 882.89 \\
\hline PSO & 254.45 & 303.12 & 406.23 & 522.19 & 647.62 & 722.45 & 882.32 \\
\hline$G A$ & 253.34 & 301.56 & 405.54 & 521.56 & 645.56 & 721.87 & 879.34 \\
\hline NRGA & & & & & & & \\
\hline
\end{tabular}


Table 6: Number of Iterations Required by Various Methods

\begin{tabular}{|c|c|c|c|}
\hline Power Demand $(M W)$ & PSO & GA & NRGA \\
\hline 400 & 120 & 91 & 65 \\
\hline 500 & 126 & 106 & 69 \\
\hline 600 & 135 & 110 & 74 \\
\hline 700 & 141 & 121 & 61 \\
\hline 800 & 136 & 119 & 71 \\
\hline 900 & 135 & 125 & 83 \\
\hline 1000 & 151 & 137 & \\
\hline
\end{tabular}

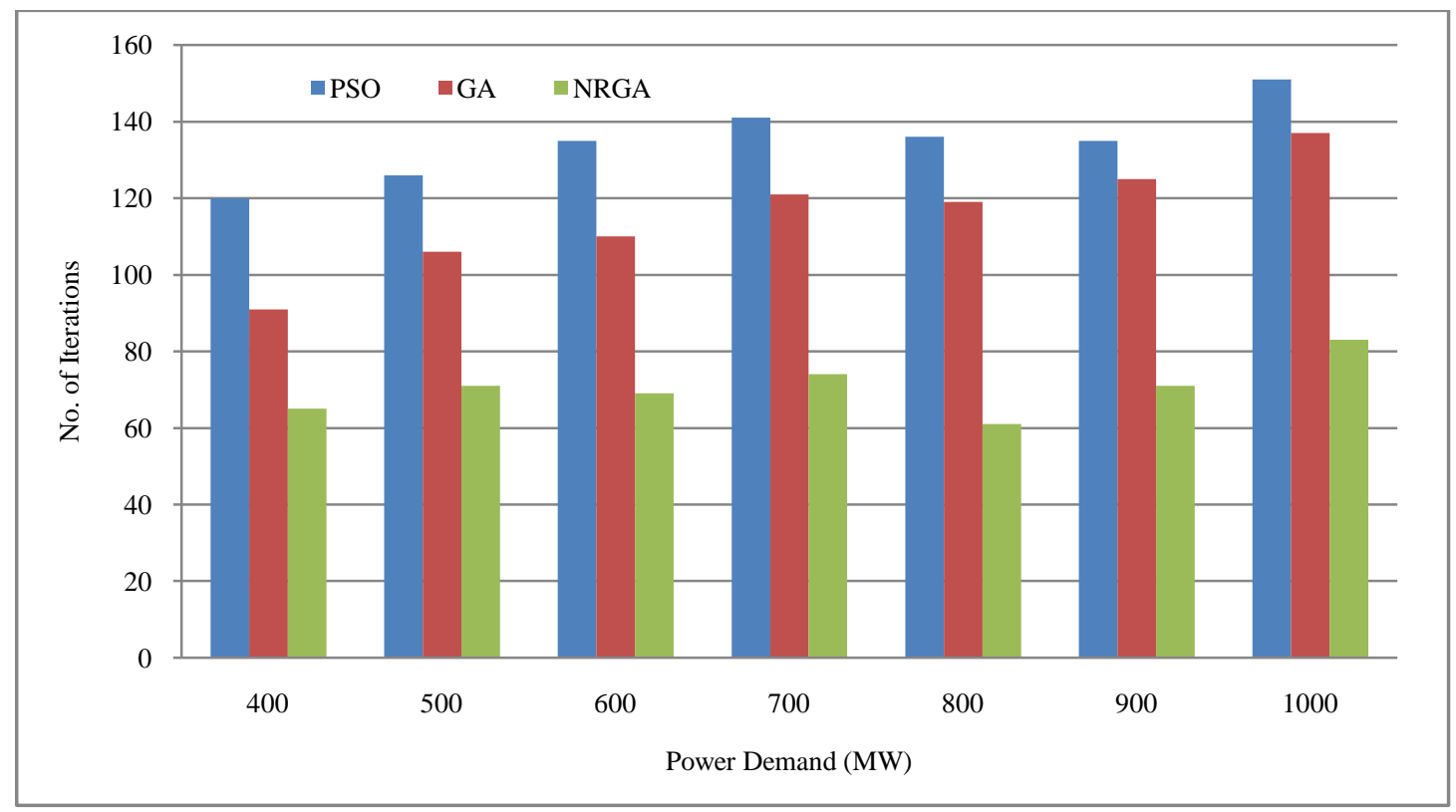

Figure 2: Comparison of Proposed Technique by means of Number of Required Iterations

Table 7: Required CPU Time (ms) by Various Methods

\begin{tabular}{|c|c|c|c|}
\hline Power Demand $(M W)$ & PSO & GA & NRGA \\
\hline 400 & 246 & 198 & 163 \\
\hline 500 & 251 & 202 & 149 \\
\hline 600 & 262 & 210 & 156 \\
\hline 700 & 234 & 187 & 161 \\
\hline 800 & 278 & 201 & 164 \\
\hline 900 & 281 & 199 & 172 \\
\hline 1000 & 298 & 215 & \\
\hline
\end{tabular}




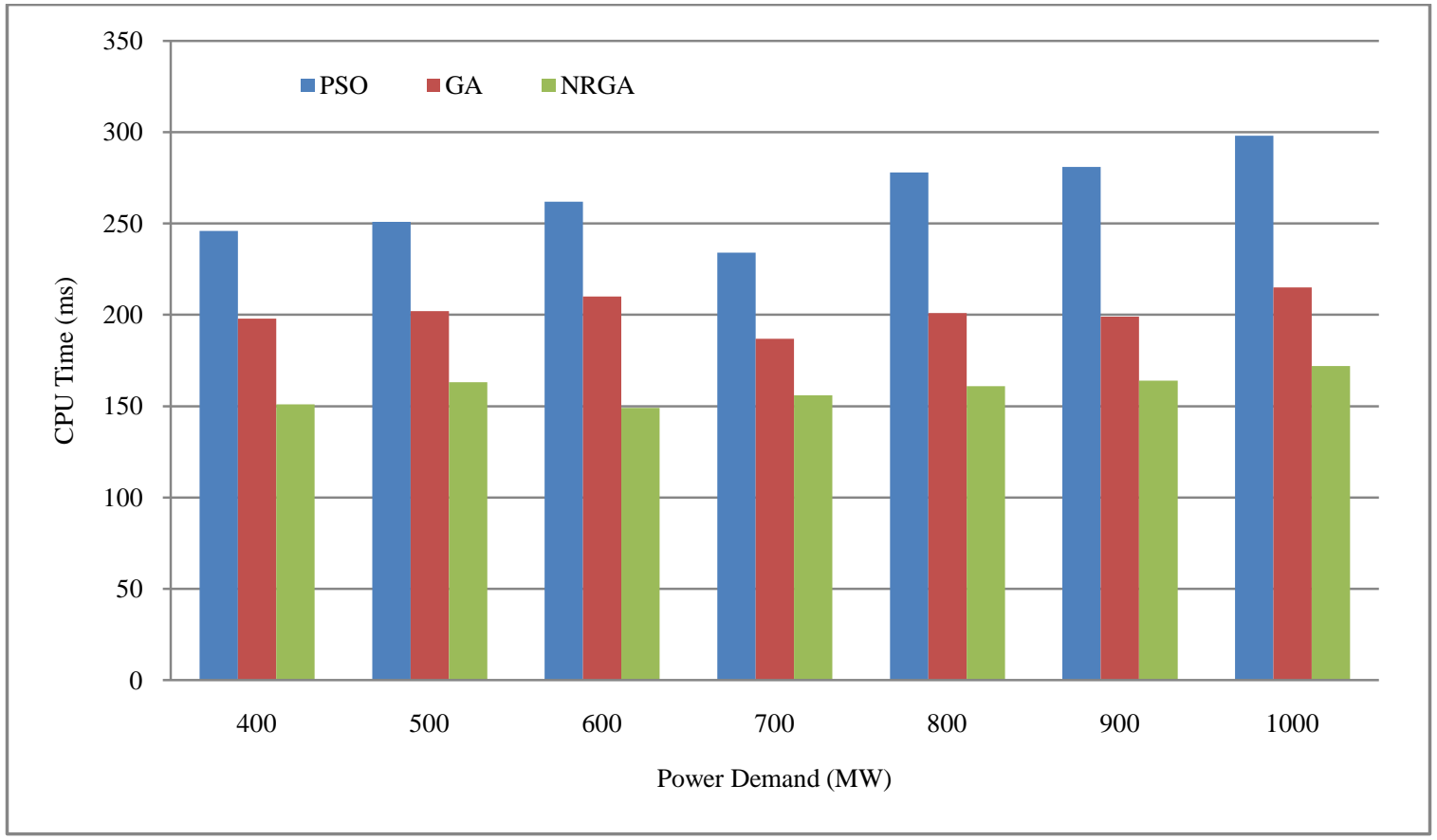

Figure 3: Comparison of Proposed Technique by means of Required CPU Time

\section{CONCLUSION}

The main intention of Economic Dispatch of electric power generation is to schedule the committed generating unit outputs so as to satisfy the load demand at minimum operating cost while fulfilling all unit and system equality and inequality constraints. By controlling the cost, the pollution causing emissions raises. Therefore, the cost reduction must be controlled by means of a technique called Emission Dispatch. But, the controlling of emission will increase the cost required for power generation. So a combined technique called Combined Economic and Emission Dispatch (CEED) emerges. There are various technique exists to deal with CEED problem which are suggested by various researchers. All the existing system has its own advantages and disadvantages. Widely used technique for CEED problem is to optimize the parameters used for power generation with the help of optimization techniques like PSO, GA, etc. But, all those techniques requires more time for optimization. To overcome those difficulties, this paper uses NRGA optimization technique. The simulation result shows the performance of the proposed technique and it can be suggested that the proposed technique reduces the fuel cost as well as the emission output. At the same time, it takes only lesser time and number of iterations for optimization.

\section{REFERENCES}

[1] S. Muralidharan, K. Srikrishna, and S. Subramanian, "A Novel ParetoOptimal Solution for Multi-Objective Economic Dispatch Problem", Iranian Journal of Electrical And Computer Engineering, Vol. 6, No. 2, 2007.
[2] Prasanna. T.S and Somasundaram. P, "Fuzzy -Tabu Search Algorithm For Combined Economic And Emission Dispatch", National Systems Conference, NSC, Pp. 17-19, 2008.

[3] Ugur Guvenc, "Combined economic emission dispatch solution using genetic algorithm based on similarity crossover", Scientific Research and Essays, Vol. 5, No.17, Pp. 2451-2456, 2010.

[4] K. Senthil and K. Manikandan, 'Improved Tabu Search Algorithm to Economic Emission Dispatch with Transmission Line Constraint", International Journal of Computer Science \& Communication, Vol. 1, No.2, Pp. 145-149, 2010.

[5] Lakshmi Devi and O. Vamsi Krishna, "Combined Economic And Emission Dispatch Using Evolutionary Algorithms-A Case Study", ARPN Journal of Engineering and Applied Sciences, Vol. 3, No. 6, 2008.

[6] Lakhwinder Singh and J.S. Dhillon, "Cardinal priority ranking based decision making for economic-emission dispatch problem", International Journal of Engineering, Science and Technology Vol. 1, No. 1, Pp. 272282, 2009.

[7] Y. Demirel and A. Demiroren, "Economic and Minimum Emission Dispatch", WSEAS Transactions on Systems, Vol. 3, No. 4, Pp. 800804, 2004.

[8] T. Ratniyomchai, A. Oonsivilai, P. Pao-la-or, and T. Kulworawanichpong, "Particle Swarm Optimization for Solving Combined Economic and Emission Dispatch Problems", Recent Advances in Energy \& Environment, 2010.

[9] M. R. Alrashidi, and M. E. El-Hawary, "Impact of Loading Conditions on the Emission- Economic Dispatch", World Academy of Science, Engineering and Technology, 2008.

[10] T. Yalcinoz, H. Altun and U. Hasan, "Environmentally Constrained Economic Dispatch via Neural Networks", 2009.

[11] K. Senthil, "Combined Economic Emission Dispatch using Evolutionary Programming Technique", IJCA Special Issue on "Evolutionary Computation for Optimization Techniques, ECOT, 2010.

[12] Vo Ngoc Dieu, Weerakorn Ongsakul, "Economic Dispatch With Emission And Transmission Constraints By Augmented Lagrange 
Hopfield Network", Transaction in Power System Optimization (GJOT), Vol.1, 2010.

[13] Ozyon S, Yasar C, Aslan, Y, Temurtas H, "Solution to environmental economic power dispatch problems in hydrothermal power systems by using genetic algorithm", International Conference on Electrical and Electronics Engineering (ELECO), Pp. 387-391, 2009.

[14] Gonggui Chen, "Combined Economic Emission Dispatch Using SFLA", International Conference on Information Engineering and Computer Science (ICIECS), Pp. 1 - 4, 2009.

[15] Arias D.A, Mota A.A, Mota L, Castro C.A, “A bilevel programming approach for power system operation planning considering voltage stability and economic dispatch", Transmission and Distribution Conference and Exposition: Latin America, 2008 IEEE/PES, Pp. 1-6, 2008.

[16] Peng Chen, Chunhua Zhao, Jian Li, Zhiming Liu, "Solving the Economic Dispatch in Power System via a Modified Genetic Particle Swarm Optimization", International Joint Conference on Computational Sciences and Optimization (CSO), Vol. 1, Pp. 201 - 204, 2009.

[17] Ravikumar Pandi V, Panigrahi B.K, Mallick M.K, Abraham A, Das S, "Improved Harmony Search for Economic Power Dispatch", Ninth International Conference on Hybrid Intelligent Systems (HIS '09), Vol. 3, Pp. 403-408, 2009.

[18] Dos Santos Coelho L, De Andrade Bernert D.L, Mariani V.C, "Chaotic differential Harmony Search algorithm applied to power economic dispatch of generators with multiple fuel options", IEEE Congress on Evolutionary Computation (CEC), Pp. 1- 5, 2010.

[19] J. Wood, B. F. Wollenberg, Power generation, operation and control, 2nd edn. New York: John Wiley \& Sons, 1996.

[20] Palanichamy and N. S. Babu, Analytical solution for combined economic and emissions dispatch, Electric Power Systems Research, Vol. 78, Pp. 1129-1137, 2008.

[21] P. Venkatesh, R. Gnanadass, and N. P. Padhy, Comparison and Application of Evolutionary Programming Techniques to Combined Economic Emission Dispatch with Line Flow Constraints, IEEE Trans. Power Syst., Vol. 18, Pp. 688-697, 2003.

[22] P. Venkatesh, K. Y. Lee, "Multi-Objective Evolutionary Programming for Economic Emission Dispatch problem", Power and Energy Society General Meeting-Conversion and Delivery of Electrical Energy in the 21st Century, Pp. 1-8, 2008.

[23] Kulkarni P.S, Kothari A.G, and Kothari D.P, "Combined Economic and Emission Dispatch Using Improved Backpropagation Neural Network", Electric Machines and Power Systems, Vol.28, Pp. 31-44, 2000.

[24] C.-L. Chiang, "Optimal economic emission dispatch of hydrothermal power systems, Electrical Power and Energy Systems", Vol.29, Pp. 462-469, 2007.

[25] M. Basu, "A simulated annealing-based goal attainment method for economic emission load dispatch of fixed head hydrothermal power systems", Electrical Power and Energy Systems, Vol. 27, Pp. 147-153, 2005.

[26] L. Wang, C. Singh, Reserve-constrained multiarea environmental/ economic dispatch based on particle swarm optimization with local search, Engineering Applications of Artificial Intelligence, Vol. 22, Pp. 298-307, 2009.

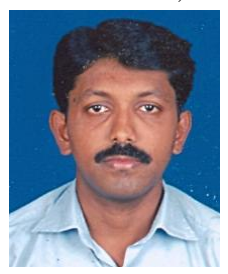

R. Gopalakrishnan, is an Assistant Professor in the Department of Electrical and Electronics Engineering at KSR College of Engineering, Tiruchengode, India. He received a B. E. in Electrical and Electronics Engineering from Bannari Amman Institute of Technology, Sathyamangalam, India in 2000, and M.E. in Power systems from Annamalai University, Chidambaram, India in 2004 and he is pursuing his $\mathrm{PhD}$ at Anna University, Chennai, India. His areas of interest include Economic and emission dispatch with Stability as constraint.

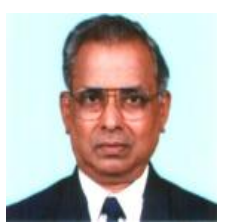

A. Krishnan, is a Dean - Academics in KSRangasamy College of Technology, Tiruchengode, India. He has received his BSc in Physics from Madras University, Chennai India in 1963, BE in Electrical Engineering from College of Engineering, Chennai, India in 1963, ME in Control Systems from PSG College of Technology, Coimbatore, India and PhD from Indian Institute of Technology, Kanpur I 1979. His research interests include Control systems and Digital systems. He has published over 60 papers in journals and technical conferences. He is a senior member of IEEE. 